\title{
Evidence for an Ultrafast Breakdown of the BeO Band Structure Due to Swift Argon and Xenon Ions
}

\author{
G. Schiwietz, ${ }^{1, *}$ K. Czerski,,${ }^{1,2}$ M. Roth, ${ }^{1}$ P. L. Grande, ${ }^{3}$ V. Koteski, ${ }^{4}$ and F. Staufenbiel ${ }^{1}$ \\ ${ }^{1}$ Helmholtz-Zentrum Berlin für Materialien und Energie, Institut G-I2, Hahn-Meitner-Platz, 1, 14109 Berlin, Germany \\ ${ }^{2}$ Instytut Fizyki, Uniwersytet Szczecinski, ul. Wielkopolska 15, 70-451 Szczecin, Poland \\ ${ }^{3}$ Universidade Federal do Rio Grande do Sul, Avenida Bento Gonçalves 9500, 91501-970 Porto Alegre, RS, Brazil \\ ${ }^{4}$ VINCA Institute, Department of Physical Chemistry, POB 522, Belgrade 11001, Serbia
}

(Received 24 February 2010; published 29 October 2010)

\begin{abstract}
Auger-electron spectra associated with Be atoms in the pure metal lattice and in the stoichiometric oxide have been investigated for different incident charged particles. For fast incident electrons, for $\mathrm{Ar}^{7+}$ and $\mathrm{Ar}^{15+}$ ions as well as $\mathrm{Xe}^{15+}$ and $\mathrm{Xe}^{31+}$ ions at velocities of $6 \%$ to $10 \%$ the speed of light, there are strong differences in the corresponding spectral distributions of Be-K Auger lines. These differences are related to changes in the local electronic band structure of $\mathrm{BeO}$ on a femtosecond time scale after the passage of highly charged heavy ions.
\end{abstract}

PACS numbers: 79.20.Rf, 31.70.Hq, 32.80.Hd, 71.20.Ps

The energy deposition of slow ions in solids may depend on details of the ion trajectory and threshold conditions are currently being investigated [1]. On the contrary, the local energy deposition of fast ions is relatively well understood and different theoretical concepts yield results that agree roughly with experiment and with each other [2,3]. The electronic energy dissipation and relaxation, however, is subject to intense investigations and especially effects of the high-energy density due to focused short lasers pulses [4] or individual swift heavy ions may give rise to unexpected results [5]. There have been speculations about a collective Coulomb explosion [6], a repulsive interaction among multiply ionized target atoms, and in fact evidence for this effect has been found for laser [4] and ion-beam interactions in some specific insulators [7]. For most materials, however, the corresponding nuclear-track potentials and fields are too low or do not last long enough to initiate materials modifications. Under these conditions, perturbative mechanisms such as the site-specific Auger-induced ionic desorption [8] or collective mechanisms driven by high kinetic electron energies may gain importance. For the latter case, either lattice instabilities [9] or electronic thermal-spike effects $[10,11]$ may play a major role, where hot electrons at electron temperatures of nearly $100000 \mathrm{~K}[12,13]$ transfer their energy to the lattice.

Usually, the energy distribution $d n\left(T_{e}, \varepsilon\right) / d \varepsilon$ of thermally equilibrated hot electrons is given by the product of total (occupied plus unoccupied) electronic density-ofstates (EDOS) $D(\varepsilon)$ and the Fermi-Dirac distribution. The resulting Auger spectrum is given by the convolution of two of such densities, $d n_{X} / d \varepsilon$ and $d n_{Y} / d \varepsilon$ for all combinations of bands $X$ and $Y$, weighted by the squared Auger matrix elements. It will be shown here, however, that Auger spectra for $\mathrm{BeO}$ are inconsistent with this simple factorization of $d n / d \varepsilon$.
The experimental setup has been described previously [5], and thus, only a short description will be given here. Electron spectra have been taken with an electrostatic electron spectrometer involving a bathtub deflection field (patent pending). The spectrometer was located at a detection angle of $135^{\circ}$ with respect to the (normal) incident ion-beam direction. Together with the sample, it was placed inside a doubly magnetically shielded ultrahigh vacuum chamber and typical residual gas pressures during the experiments were close to $10^{-10}$ mbar. Specifically for the experiments with $\mathrm{Be}$ or $\mathrm{BeO}$ targets, the channel-plate detector was shielded by a Ta block to reduce the count rate due to nuclear reactions strongly.

As shown previously [14], thin $\mathrm{BeO}$ films of about $3 \mathrm{~nm}$ thickness [15] have been prepared by implanting $500 \mathrm{eV}$ oxygen ions into atomically clean Be (preventing also macroscopic surface changing). These films are completely eroded within one or two hours of ion irradiation, as we may conclude from the change of Auger-electron spectra as well as from secondary-ion spectra [14]. The elevated global target temperature of about $200{ }^{\circ} \mathrm{C}$ during ion irradiation may lead to enhanced evaporation. In the final ion-beam times presented here, we have used either pure oxygen exposure at about $10^{-6}$ mbar or alternatively continuous $500 \mathrm{eV}$ O-ion implantation inside the main chamber, in order to prevent any net erosion effect. In both cases, we found time-independent Auger spectra for several hours of ion irradiation as well as for electron irradiation, pointing to dynamically clean and stable surface structures.

Experiments have been performed using $\mathrm{Ar}^{7+}$ ions at a specific kinetic energy of $3 \mathrm{MeV} /$ nucleon as well as with $1.78 \mathrm{MeV} / \mathrm{u} \mathrm{Xe}^{15+}$, both provided directly by the cyclotron of the ion-beam laboratory Berlin. Charge equilibrated $\mathrm{Ar}^{15+}$ ions and $\mathrm{Xe}^{31+}$ ions have been extracted behind a thin $\mathrm{C}$ stripper foil in front of the target (this 
suppresses depth dependencies due to charge equilibration [5]) and electrons at $2.7 \mathrm{keV}$ have also been used as projectiles. Although the fast projectiles penetrate deep inside the target, the emitted electrons investigated in this work stem only from the first few surface layers (Be $K$-Auger electrons have an inelastic mean-free-path below $1 \mathrm{~nm}$ ) [16]. In between the ion-beam experiments, Auger spectra induced by primary electrons were taken periodically to make sure that the sample composition did not change.

For this purpose, the electron beam (spot size $\ll 1 \mathrm{~mm}$ ) was placed on the target in the center of the ion spot (diameter about $2 \mathrm{~mm}$ ), providing also a precise and reproducible energy scale for all spectra. This alignment was performed by focusing each of the beams through the same small aperture $(\varnothing 2.3 \mathrm{~mm}$ and distance to the target center of $26.4 \mathrm{~mm}$ ) in the target plane and subsequent precision translation of the target onto exactly this point in space (manipulator precision $<10 \mu \mathrm{m}$ ). The beam overlap was proven by ion-induced surface cleaning as well as materials modifications [5].

Our target preparation results in stoichiometric amorphous $\mathrm{BeO}(a-\mathrm{BeO})$ as indicated by simulation of the spectrum [16] and especially as proven by comparison of our Auger data with different literature sources. Such a comparison is shown in Fig. 1 for $a-\mathrm{BeO}$ films from reactive (using an $\mathrm{Ar} / \mathrm{O}_{2}$ gas mixture) rf sputter deposition onto clean metallic $\mathrm{Be}$ [17], from exposure of a clean $\mathrm{Be}$ surface to oxygen gas [18] as well as for a single crystal $\mathrm{BeO}$ bulk sample after ion sputter cleaning of the surface [19]. The plot shows differentiated energy spectra (a typical presentation for analytical purposes), not corrected for the different spectrometer transmission functions. In one

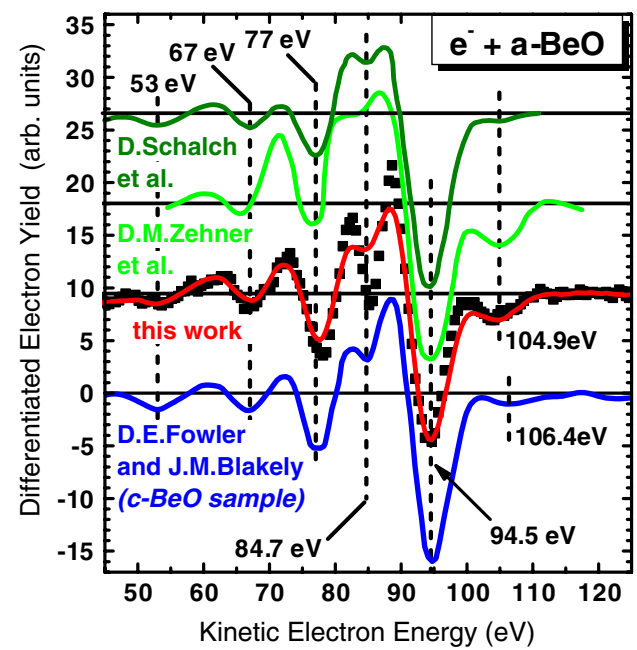

FIG. 1 (color online). Differentiated Auger-electron spectra. The black symbols and smoothed (red) curve were obtained in this work (averaged over 18 spectra induced by $2.7 \mathrm{keV}$ electrons during the heavy-ion beam time). Other curves are taken from the literature and correspond also to $a-\mathrm{BeO}[17,18]$ as well as to a $\mathrm{BeO}$ bulk single crystal $(c-\mathrm{BeO})$ [19]. case [18] a linear background subtraction and in two other cases $[17,19]$ sub-eV energy-scale corrections have been performed to improve the overlap among the different spectra.

It can be seen that all spectra are very similar, although they have been taken under different experimental conditions. Two significant deviations, however, may be seen in the plot. Firstly, the spectra taken in this work (black squares) show a more pronounced dip structure around $85 \mathrm{eV}$, but the spectral shape becomes very similar to the other three spectra when our data set is artificially broadened $(\Delta E / E=5 \%$ instead of $1 \%)$ resulting in the (red) curve that crosses the data points. Secondly, the lowest curve for $c$-BeO has a high-energy dip (at $106.4 \mathrm{eV}$ ), $1.5 \mathrm{eV}$ above the corresponding dip in the $a$ - $\mathrm{BeO}$ spectra. This dip might be due to core-hole excitons [20], slightly below the conduction band. The energy shift is consistent with the fact that the band gap in $c-\mathrm{BeO}$ exceeds that of $a$-BeO by about $3 \mathrm{eV}$.

In addition to our experimental approach, we have also performed supercell calculations using the well-known WIEN solid-state code [21] for a $\mathrm{Be}^{+}$ion (with a $K$-shell vacancy) inside the atomically frozen $\mathrm{BeO}$ (Wurtzite) structure. These calculations show (in comparison to results without a localized $K$-shell vacancy) the existence of core-hole excitons, consistent with the dip at high energy as well as the weak one at $85 \mathrm{eV}$. Quadratic scaling of the transition rates with the local Be valence-electron densities (including $L_{1}$ and for a $K$ vacancy) have been used to estimate the $\mathrm{Be}-\mathrm{K}$ decay-time $\tau_{\text {Auger }} \approx 300 \mathrm{fs}$ for $\mathrm{BeO}$ from the known value of the pure $\operatorname{Be}$ metal $\left(\tau_{\text {Auger }} \approx\right.$ 15 fs [22]). From the EDOS and from auxiliary electron energy-loss measurements at primary energies around $100 \mathrm{eV}$, we have estimated the resulting Auger-electron spectrum (to be published in a forthcoming paper). Using this information, the dominant structures at $94.5,77$, and $67 \mathrm{eV}$ in Fig. 1 may be assigned to the $K-V V, K-L_{1} V$ and $K-L_{1} L_{1}$ Auger transitions (involving the full valence band $V$ and the narrow $L_{1}$ band).

After the above discussion of the electron-induced spectrum (small perturbation of the solid), we focus now on the strong perturbations that are produced by highly charged ions at similar velocities (at specific kinetic energies of a few $\mathrm{MeV} / \mathrm{u}$ ). Figure 2 displays Be $K-X V$ Auger electron spectra $\left(X=L_{1}\right.$ or $\left.V\right)$ obtained in this work for $a-\mathrm{BeO}$ and induced by very different projectiles. The solid black curve in Fig. 2 is due to $2.7 \mathrm{keV}$ incident electrons and it is reasonably well understood, leading after differentiation to the black squares in Fig. 1. The other curves and plot symbols in Fig. 2 are due to swift heavy ions, such as $3 \mathrm{MeV} / \mathrm{u} \mathrm{Ar}^{7+}$ and $\mathrm{Ar}^{15+}$ (dark cyan and green curves with triangular symbols) and in addition $1.78 \mathrm{MeV} / \mathrm{u}$ $\mathrm{Xe}^{15+}$ and $\mathrm{Xe}^{31+}$ ions (orange and red curves with crosses). Several variations of the spectra may be noted when going from low charge states (incident electrons) to the highest charge states ( $\mathrm{Xe}^{31+}$ ions). 


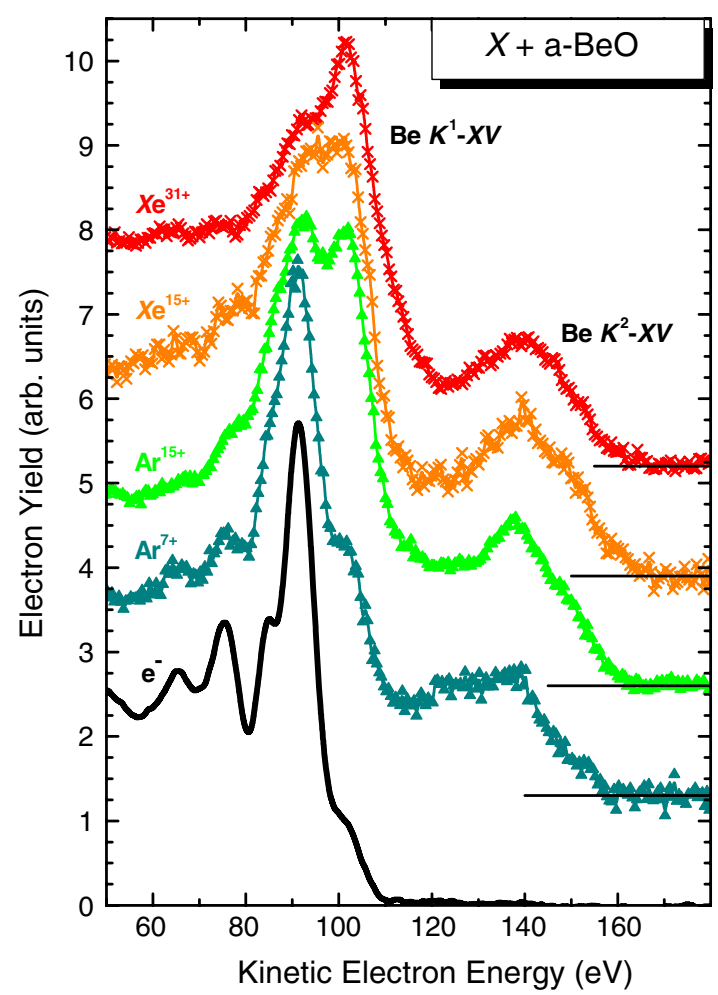

FIG. 2 (color online). Projectile dependence of the $\mathrm{Be}$ $K$-Auger electron spectrum for $a$-BeO due to fast $\mathrm{Ar}$ and $\mathrm{Xe}$ ions of different incident charge states (averaged over many raw spectra) and $2.7 \mathrm{keV}$ electrons. Secondary-electron backgrounds have been subtracted and intensity offsets have been applied, as indicated by the base lines on the right side.

First, a strong and broad peak emerges for heavy ions at electron energies above $120 \mathrm{eV}$. This peak is due to Auger decay $\left(\mathrm{Be} K^{2}-X V\right)$ of Be double $K$-shell vacancies and is a typical indication of strong perturbations [5]. The nearly constant intensity ratio $N\left(K^{2}-X V\right) / N\left(K^{1}-X V\right)$ observed for heavy ions corresponds to a $15 \%$ fraction of double $K$ ionization of Be (after correcting for Auger cascades, for the electron escape length, and for the spectrometer transmission [5]). This constant fraction results from the limitation of inner-shell reactions to a maximum probability of $100 \%$ at small impact parameters [5,12]. Each spectrum reflects a snapshot of the time evolution of electrons close to the Be vacancy, averaged over the corresponding decay time $\tau_{\text {Auger }}$. For the cold system (primary electrons), the Auger-decay time is expected to reflect the low electron density of $\mathrm{Be}$ in the ionic compound, but high densities might be reached for an extremely hot target (for highly charged projectiles).

In contrast to all previous investigations for other materials (metals, semimetals, semiconductors, and polymers) [5,12,13]; however, the $K^{1}-X V$ peak structure in Fig. 2 is strongly varying as function of the projectile. We find a broadening of the peak structure below $92 \mathrm{eV}$. Further, there is a growing peak for highly charged ions superimposed roughly at the position of the core-hole exciton peak at $101 \mathrm{eV}$ (a detailed fit indicates that the new peak lies about $1 \mathrm{eV}$ higher). Comparable charge-dependent variations of solid-state Auger spectra have not been found before for any material so far $[5,12,13]$. Most likely, the changes of the peak are related to a breakdown of the $\mathrm{BeO}$ band structure on a femtosecond time scale, as will be discussed in the following.

Heavy-ion tracks are accompanied by many hot electrons and electron temperatures of up to $10^{5} \mathrm{~K}$ have experimentally been determined from the broadening of the high-energy profile of Auger lines, e.g., for individual ions in pure metallic Be [12]. For a fixed EDOS, the charge-dependent broadening of Auger lines is related to a temperature-dependent broadening of the FermiDirac distribution $f\left(T_{e}, \varepsilon\right)$. The distribution $f\left(T_{e}, \varepsilon\right)$ may, e.g., lower the peak intensity at $92 \mathrm{eV}$ and increase the one at $102 \mathrm{eV}$, but this would correspond to unrealistically high electron temperatures exceeding several $10^{5} \mathrm{~K}$. Such high $T_{e}$ values would be accompanied by very broad high-energy features due to states leading to Auger structures even in excess of $110 \mathrm{eV}$. Within this standard picture, it is impossible that there is an abrupt variation of a single line intensity for $\mathrm{Ar}^{7+}$ compared to $\mathrm{Ar}^{15+}$ ions in Fig. 2, without the appearance of additional $K^{1}-X V$ structures at still higher energies. This means that the variation of the spectra in Fig. 2 cannot simply be related to a Fermi-Dirac distribution multiplied with an EDOS from a frozen band structure.

Materials modifications due to either the nuclear energy loss or the extremely high electronic energy loss of heavy ions could, in principle, change the EDOS. But our electroninduced spectra (taken in between the series of ion runs) as well as the ion-induced spectra point to steady-state conditions on a time scale of minutes (individual Auger spectra) or even much less (extracted from target currents measured for each channel and from ion-desorption spectra [14] taken previously) up to several hours (time between target preparations), thereby excluding permanent materials modifications [23]. Correspondingly, the change of the spectra in Fig. 2 should be due to an intrinsic effect, related to individual collisions. This points to a breakdown of the initial-state band-structure on the time scale of the $\mathrm{Be}$ $K$-Auger transition or faster. The question about the basic cause of this breakdown and why it has not been seen before, will be discussed in the following.

The high degree of electronic excitation may easily lead to a nonequilibrium electron distribution where electrons are largely found in excited states, not restricted to the oxygen lattice sites, and the ionic character of $\mathrm{BeO}$ is lost (similar to the modified charge distribution in the KnotekFeibelman process [8]). Such a state would be specific for fast heavy ions in ionic compounds, a case not investigated with high resolution Auger-electron spectroscopy so far (most previous Auger-electron investigations in the electronic-stopping regime have been performed with light ions at lower velocities and only for monoatomic targets [24]). This nonionic electron density may lead to 


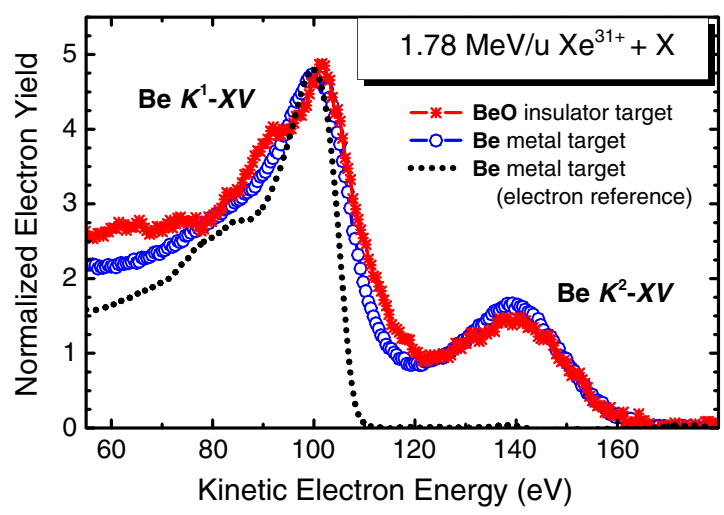

FIG. 3 (color online). Comparison of the Be $K$ Auger-electron spectra (after background subtraction) for $a$-BeO with clean metallic $\mathrm{Be}$, both excited by $230 \mathrm{MeV}{ }^{129} \mathrm{Xe}^{31+}$ ions. A reference spectrum for metallic Be ionized by incident electrons and averaged over the two primary energies of 1.0 and $2.7 \mathrm{keV}$ (without any significant difference) is also shown.

Auger spectra as in Fig. 2 through (i) modified electronic screening and correlation effects or (ii) modified atomic interaction potentials that may lead to a (rapid) cold melting of the lattice [25].

We cannot distinguish between these two scenarios, but Fig. 3 indicates that $\mathrm{BeO}$ has reached a metal-like state, independent of a specific scenario (i) or (ii). This plot shows the Auger spectrum corresponding to the cold metallic state of $\mathrm{Be}$ (after electron-induced ionization) as well as two spectra for $\mathrm{Xe}^{31+}$ ions on $\mathrm{Be}$ and on $\mathrm{BeO}$. Both ion-induced spectra are very similar, with a comparable energy position and width of the peak structure (these widths may be converted into reasonable electron temperature values of about $55000 \mathrm{~K}[5,12])$. This similarity is an indication that we do not only see the hot metallic state for the Be target [12], but for the excited $\mathrm{BeO}$ target as well.

In summary, our experimental determinations of emitted Be target Auger-electron spectra induced by fast heavy ions provide the first illustration of a breakdown or instability of the electronic band structure of $\mathrm{BeO}$ at higher electron temperatures. Auger-electron spectra for incident ions deviate drastically from those induced by primary electrons under otherwise similar experimental conditions. Moreover, comparison of heavy-ion-induced $\mathrm{BeO}$ with pure Be target Auger-electron spectra shows very similar spectral shapes. We take this as an indication of a temporary metal-like reconfiguration of the electronic levels in $\mathrm{BeO}$ triggered by strong electronic excitations. One might speculate that this is a signature of an ultrafast melting of the lattice, but the corresponding conclusion can only be drawn with further information.

We are indebted to the crew of the former ISL cyclotron for providing stable and intense ion beams and we acknowledge funding by the Alexander von Humboldt foun- dation, by $\mathrm{CNPq}$, and last but not least, many important comments by $\mathrm{S}$. Klaumünzer.

*Corresponding author: schiwietz(at)helmholtz-berlin.de

[1] S. N. Markin, D. Primetzhofer, and P. Bauer, Phys. Rev. Lett. 103, 113201 (2009).

[2] G. de M. Azevedo et al., Phys. Rev. Lett. 86, 1482 (2001); G. Schiwietz et al., Phys. Rev. Lett. 72, 2159 (1994).

[3] A. Schinner and P. Sigmund, Nucl. Instrum. Methods Phys. Res., Sect. B 195, 64 (2002).

[4] R. Stoian et al., Phys. Rev. Lett. 88, 097603 (2002).

[5] G. Schiwietz et al., Nucl. Instrum. Methods Phys. Res., Sect. B 226, 683 (2004).

[6] R. L. Fleischer, P. B. Price, and R. M. Walker, Nuclear Tracks in Solids (University of California Press, Berkeley, California, 1975).

[7] G. Xiao et al., Phys. Rev. Lett. 79, 1821 (1997).

[8] M. L. Knotek and P. J. Feibelman, Phys. Rev. Lett. 40, 964 (1978).

[9] P. B. Hillyard et al., Phys. Rev. Lett. 98, 125501 (2007).

[10] Z. G. Wang, C. Dufour, E. Paumier, and M. Toulemonde, J. Phys. Condens. Matter 6, 6733 (1994).

[11] K. Sokolowski-Tinten et al., Phys. Rev. Lett. 81, 224 (1998).

[12] G. Schiwietz et al., Europhys. Lett. 47, 384 (1999); F. Staufenbiel et al., Nucl. Instrum. Methods Phys. Res., Sect. B 230, 426 (2005).

[13] M. Caron, H. Rothard, M. Beuve, and B. Gervais, Phys. Scr. T92, 281 (2001).

[14] K. Czerski et al., Nucl. Instrum. Methods Phys. Res., Sect. B 225, 72 (2004).

[15] Estimated using the SRIM-2008 code; by J. F. Ziegler, J. P. Biersack, and U. Littmark, The Stopping and Range of Ions in Solids (Pergamon Press, New York, 2009).

[16] NIST Ref. Database 100 SESSA 1.1; W. Smekal, W. S. M. Werner, and C. J. Powell, Surf. Interface Anal. 37, 1059 (2005).

[17] D. Schalch, A. Scharmann, and A. Weiß, Thin Solid Films 124, 351 (1985).

[18] D. M. Zehner, N. Barbulesco, and L. H. Jenkins, Surf. Sci. 34, 385 (1973).

[19] D. E. Fowler and J. M. Blakely, J. Vac. Sci. Technol. 20, 930 (1982).

[20] J. A. Soininen et al., J. Phys. Condens. Matter 13, 8039 (2001).

[21] P. Blaha, K. Schwarz, and J. Luitz, WIEN97 (Karlheinz Schwarz, Technische Universität Wien, Austria, 1999) ISBN 3-9501031-0-4.

[22] M. Krause and J. H. Oliver, J. Phys. Chem. Ref. Data 8, 329 (1979).

[23] An independent argument against any influence of materials modifications is the observed insensitivity regarding variations of the primary-ion flux.

[24] D. Hasselkamp, Particle Induced Electron Emission II, Springer Tracts of Modern Physics, Vol. 123 (Springer, Berlin, 1991), and references therein.

[25] P. L. Silvestrelli, M. Parrinello, J. Appl. Phys. 83, 2478 (1998), and references therein. 